\title{
Constructing an Interactive Natural Language Interface for Relational Databases.
}

\author{
Fei Li \\ Univ. of Michigan, Ann Arbor \\ lifei@umich.edu
}

\author{
H. V. Jagadish \\ Univ. of Michigan, Ann Arbor \\ jag@umich.edu
}

\begin{abstract}
Natural language has been the holy grail of query interface designers, but has generally been considered too hard to work with, except in limited specific circumstances. In this paper, we describe the architecture of an interactive natural language query interface for relational databases. Through a carefully limited interaction with the user, we are able to correctly interpret complex natural language queries, in a generic manner across a range of domains. By these means, a logically complex English language sentence is correctly translated into a SQL query, which may include aggregation, nesting, and various types of joins, among other things, and can be evaluated against an RDBMS. We have constructed a system, NaLIR (Natural Language Interface for Relational databases), embodying these ideas. Our experimental assessment, through user studies, demonstrates that NaLIR is good enough to be usable in practice: even naive users are able to specify quite complex ad-hoc queries.
\end{abstract}

\section{INTRODUCTION}

Querying data in relational databases is often challenging. SQL is the standard query language for relational databases. While expressive and powerful, SQL is too difficult for users without technical training. Even for users with expertise in programming languages, it can be challenging because it requires that users know the exact schema of the database, the roles of various entities in a query, and the precise join paths to be followed. As the database user base is shifting towards non-experts, designing user-friendly query interfaces will be a more important goal in database community.

In the real world, people ask questions in natural language, such as English. Not surprisingly, a natural language interface is regarded by many as the ultimate goal for a database query interface, and many natural language interfaces to databases (NLIDBs) have been built towards this goal $[2,13,11]$. NLIDBs have many advantages over other

\section{*Supported in part by NSF grants IIS 1250880 and IIS 1017296}

This work is licensed under the Creative Commons AttributionNonCommercial-NoDerivs 3.0 Unported License. To view a copy of this license, visit http://creativecommons.org/licenses/by-nc-nd/3.0/. Obtain permission prior to any use beyond those covered by the license. Contact copyright holder by emailing info@vldb.org. Articles from this volume were invited to present their results at the 41st International Conference on Very Large Data Bases, August 31st - September 4th 2015, Kohala Coast, Hawaii.

Proceedings of the VLDB Endowment, Vol. 8, No. 1

Copyright 2014 VLDB Endowment 2150-8097/14/09. widely accepted query interfaces (keyword-based search, formbased interface, and visual query builder). For example, a typical NLIDB would enable naive users to specify complex, ad-hoc query intent without training. In contrast, keywords are insufficient to convey complex query intent, form-based interfaces can be used only when queries are predictable and limited to the encoded logic, and visual query builders still requires extensive schema knowledge of the user.

Despite these advantages, NLIDBs have not been adopted widely. The fundamental problem is that understanding natural language is hard. People may use slang words, technical terms, and dialect-specific phrasing, none of which may be known to the system. Even without these, natural language is inherently ambiguous. Even in human-to-human interaction, there are miscommunications. Therefore, we cannot reasonably expect an NLIDB to be perfect. Therefore, users may be provided with a wrong answer due to the system incorrectly understanding or handling the query. The system does not help user detect such error and sometimes it is impossible for users to verify the answer by themselves. So a user cannot be sure that the answer provided is really the answer to the question asked. Moreover, even if the user does realize that the answer is wrong, there is little guidance on what to do. The only option available to the user is to rephrase the query and hope that the system now understands it better.

When humans communicate with one another in natural language, the query-response cycle is not as rigid as in a traditional database system. If a human is asked a query that she does not understand, she will seek clarification. She may do so by asking specific questions back, so that the question-asker understands the point of potential confusion. She may also do so by stating explicitly how she interpreted the query. Drawing inspiration from this natural human behavior, we design the query mechanism to facilitate collaboration between the system and the user in processing natural language queries. First, the system explains how it interprets a query, from each ambiguous word/phrase to the meaning of the whole sentence. These explanations enable the user to verify the answer and to be aware where the system misinterprets her query. Second, for each ambiguous part, we provide multiple likely interpretations for the user to choose from. Since it is often easier for users to recognize an expression rather than to compose it, we believe this query mechanism can achieve satisfactory reliability without burdening the user too much.

A question that then arises is how should a system represent and communicate its query interpretation to the user. 


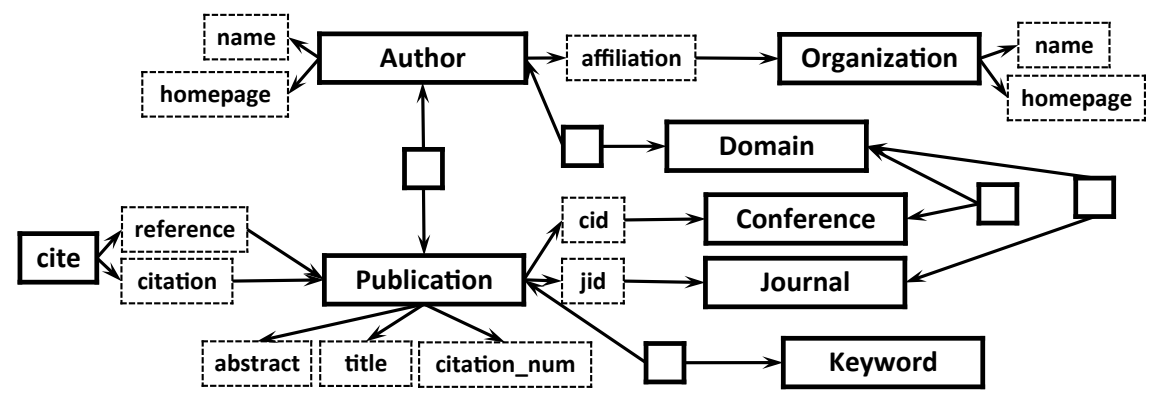

Query 1: Return the average number of publications by Bob in each year.

Query 2: Return authors who have more papers than Bob in VLDB after 2000.

Query 3: Return the conference in each area whose papers have the most total citations.

Figure 1: A Simplified Schema for Microsoft Academic Search and Sample Queries.

SQL is too difficult for most non-technical humans. We need a representation that is both "human understandable" and "RDBMS understandable". In this paper, we present a data structure, called Query Tree, to meet this goal. As an intermediate between a linguistic parse tree and a SQL statement, a query tree is easier to explain to the user than a SQL statement. Also, given a query tree verified by the user, the system will almost always be able to translate it into a correct SQL statement.

Putting the above ideas together, we propose an NLIDB comprising three main components: a first component that transforms a natural language query to a query tree, a second component that verifies the transformation interactively with the user, and a third component that translates the query tree into a SQL statement. We have constructed such an NLIDB, and we call it a NaLIR (Natural Language Interface to Relational databases).

The intellectual contributions of this paper are as follows:

1. Interactive Query Mechanism. We design an interaction mechanism for NLIDBs to enable users to ask complex queries and have them interpreted correctly, with a little interaction help.

2. Query Tree. We design a query tree structure to represent the interpretation of a natural language query from the database's perspective. A query tree can be explained to the user for verification, and once verified, will almost always be correctly translated to SQL.

3. System Architecture. We provide a modular architecture to support such a query mechanism, in which each component can be designed, and improved, independently. We develop a working software system called NaLIR, instantiating this architecture. We discuss the basic ideas in designing heuristic functions for each component and describe the specific choices made in our system.

4. User Study. We demonstrate, through carefully designed user studies, that NaLIR is usable in practice, on which even naive users are able to handle quite complex ad-hoc query tasks.

The remaining parts of the paper are organized as follows. We discuss the query mechanism in Section 2. The system architecture of our system is described in Section 3. Given a query, we show how to interpret each its words/phrases in Section 4 and infer the semantic meaning of the whole query (represented by a query tree) in Section 5. We discuss how to translate a query tree to a SQL statement in Section 6 . In Section 7, our system is evaluated experimentally. We discuss related work in Section 8. In Section 9, we draw conclusions and point to future work.

\section{QUERY MECHANISM}

Keyword search systems are popular and effective in at least some domains, such as for document search. As we think about the architecture of an NLIDB, it is worthwhile to draw inspiration from search engines, and how they infer user intent from limited information. First, given a query, a search engine returns a list of results, rather than a single result. This is central to providing acceptable recall. Second, users are able to verify whether a result is correct (useful) by reading the abstract/content. Third, these results are well ranked, to minimize user burden to verify potential answers. These strategies work very well in search engines. However, due to some fundamental differences between search engines and NLIDBs, as we will discuss next, this query mechanism cannot be directly applied to NLIDBs.

First, users are often able to verify the results from a search engine by just reading the results. However, the results returned by an NLIDB cannot usually explain themselves. For example, suppose a user submits Query 1 in Figure 1 to an NLIDB and gets an answer "5". How could she verify whether the system understands her query and returns to her the correct answer? To facilitate this, an NLIDB should provide explanations for the result at least in terms of how the query is processed.

Second, unlike search engines, users tend to express sophisticated query logics to an NLIDB and expect perfect results. That requires the NLIDB to fix all the ambiguities and get a perfect interpretation for the query from the perspective of both linguistics and the given database. However, natural language queries are often inherently ambiguous and sometimes, some of the ambiguities are too "hard" for systems to fix with confidence. Consider Query 1 in Figure 1 again, the user specifies the word "publication", while in a real world bibliography database, the information of publications may be stored in many tables, say, article, book, incollection, phdThesis, journal, proceedings and so forth. The system itself cannot be expected to figure out which 


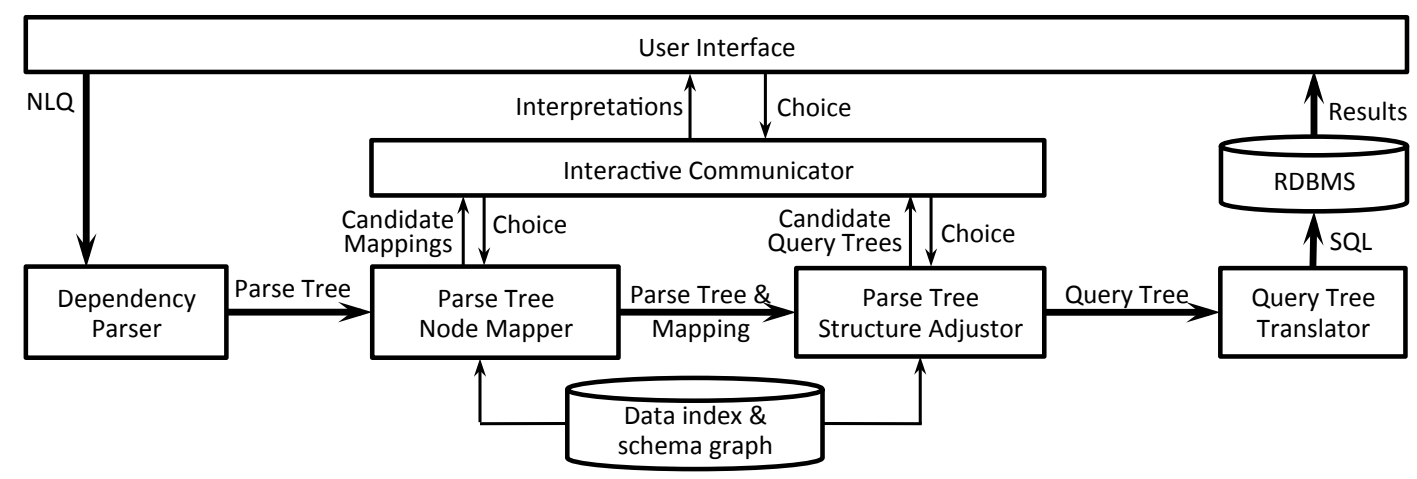

Figure 2: System Architecture.

ones of these should be considered as publications. Even if the included items are all unambiguous, when natural language queries have complex structures with modifier attachments, aggregations, comparisons, quantifiers, and conjunctions, it may contain several ambiguities that cannot be fixed by the system with confidence, from the interpretation of an ambiguous phrase to the relationship between words/phrases. The number of possible interpretations grows exponentially with the number of unfixed ambiguities. As a result, there may be hundreds of candidate interpretations for a complex natural language query, of which only one is correct. Since these interpretations are often similar to each other in semantics, it is very hard to develop an effective ranking function for them. As a result, if the system simply returns a list of hundreds of answers, the users will be frustrated in verifying them.

Given the above two observations, instead of explaining the query results, we explain the query interpretation process, especially how each ambiguity is fixed, to the user. In our system, we fix each "easy" ambiguity quietly. For each "hard" ambiguity, we provide multiple interpretations for the user to choose from. In such a way, even for a rather complex natural language query, verifications for 3-4 ambiguities is enough, in which each verification is just making choices from several options.

The ambiguities in processing a natural language query are not often independent of each other. The resolution of some ambiguities depends on the resolution of some other ambiguities. For example, the interpretation of the whole sentence depends on how each of its words/phrases is interpreted. So the disambiguation process and the verification process should be organized in a few steps. In our system, we organize them in three steps, as we will discuss in detail in the next section. In each step, for a "hard" ambiguity, we generate multiple interpretations for it and, at the same time, use the best interpretation as the default choice to process later steps. Each time a user changes a choice, our system immediately reprocesses all the ambiguities in later steps and updates the query results.

\section{SYSTEM OVERVIEW}

Figure 2 depicts the architecture of $\mathrm{NaLIR}^{1}$. The entire system we have implemented consists of three main parts:

${ }^{1}$ In the current implementation, we use MySQL as the RDBMS, and Stanford NLP Parser [7] as the dependency natural language parser. the query interpretation part, interactive communicator and query tree translator. The query interpretation part, which includes parse tree node mapper (Section 4) and structure adjustor (Section 5), is responsible for interpreting the natural language query and representing the interpretation as a query tree. The interactive communicator is responsible for communicating with the user to ensure that the interpretation process is correct. The query tree, possibly verified by the user, will be translated into a SQL statement in the query tree translator (Section 6) and then evaluated against an RDBMS.

Dependency Parser. The first obstacle in translating a natural language query into a SQL query is to understand the natural language query linguistically. In our system, we use the Stanford Parser [7] to generate a linguistic parse tree from the natural language query. The linguistic parse trees in our system are dependency parse trees, in which each node is a word/phrase specified by the user while each edge is a linguistic dependency relationship between two words/phrases. The simplified linguistic parse tree of Query 2 in Figure 1 is shown in Figure 3 (a).

Parse Tree Node Mapper. The parse tree node mapper identifies the nodes in the linguistic parse tree that can be mapped to SQL components and tokenizes them into different tokens. In the mapping process, some nodes may fail in mapping to any SQL component. In this case, our system generates a warning to the user, telling her that these nodes do not directly contribute in interpreting her query. Also, some nodes may have multiple mappings, which causes ambiguities in interpreting these nodes. For each such node, the parse tree node mapper outputs the best mapping to the parse tree structure adjustor by default and reports all candidate mappings to the interactive communicator.

Parse Tree Structure Adjustor. After the node mapping (possibly with interactive communications with the user), we assume that each node is understood by our system. The next step is to correctly understand the tree structure from the database's perspective. However, this is not easy since the linguistic parse tree might be incorrect, out of the semantic coverage of our system or ambiguous from the database's perspective. In those cases, we adjust the structure of the linguistic parse tree and generate candidate interpretations (query trees) for it. In particular, we adjust the structure of the parse tree in two steps. In the first step, we reformulate the nodes in the parse tree to make it fall in the syntactic coverage of our system (valid parse tree). If 
there are multiple candidate valid parse trees for the query, we choose the best one as default input for the second step and report top $k$ of them to the interactive communicator. In the second step, the chosen (or default) valid parse tree is analyzed semantically and implicit nodes are inserted to make it more semantically reasonable. This process is also under the supervision of the user. After inserting implicit nodes, we obtain the exact interpretation, represented as a query tree, for the query.

Interactive Communicator. In case the system possibly misunderstands the user, the interactive communicator explains how her query is processed. In our system, interactive communications are organized in three steps, which verify the intermediate results in the parse tree node mapping, parse tree structure reformulation, and implicit node insertion, respectively. For each ambiguous part, we generate a multiple choice selection panel, in which each choice corresponds to a different interpretation. Each time a user changes a choice, our system immediately reprocesses all the ambiguities in later steps.

ExAmple 1. Consider the linguistic parse tree $T$ in Figure $3(a)$. In the first step, the parse tree node mapper generates the best mapping for each node (represented as $M$ and shown in Figure $3(b))$ and reports to the user that the node "VLDB" maps to "VLDB conference" and "VLDB Journal" in the database and that our system has chosen "VLDB conference" as the default mapping. According to $M$, in the second step, the parse tree adjustor reformulates the structure of $T$ and generates the top $k$ valid parse trees $\left\{T_{i}^{M}\right\}$, in which $T_{1}^{M}$ (Figure $3(\mathrm{c})$ ) is the best. The interactive communicator explains each of the $k$ valid parse trees in natural language for the user to choose from. For example, $T_{1}^{M}$ is explained as "return the authors, where the papers of the author in VLDB after 2000 is more than Bob". In the third step, $T_{1}^{M}$ is fully instantiated in the parse tree structure adjustor by inserting implicit nodes (shown in Figure $3(d)$ ). The result query tree $T_{11}^{M}$ is explained to the user as "return the authors, where the number of papers of the author in $V L D B$ after 2000 is more than the number of paper of Bob in VLDB after 2000.", in which the underline part can be canceled by the user. When the user changes the mapping strategy $M$ to $M^{\prime}$, our system will immediately use $M^{\prime}$ to reprocess the second and third steps. Similarly, if the user choose $T_{i}^{M}$ instead of $T_{1}^{M}$ as the best valid parse tree, our system will fully instantiate $T_{i}^{M}$ in the third step and update the interactions.

Query Tree Translator. Given the query tree verified by the user, the translator utilizes its structure to generate appropriate structure in the SQL expression and completes the foreign-key-primary-key (FK-PK) join paths. The result SQL statement may contain aggregate functions, multi-level subqueries, and various types of joins, among other things. Finally, our system evaluates the translated SQL statement against an RDBMS and returns the query results back to the user.

\section{PARSE TREE NODE INTERPRETATION}

To understand the linguistic parse tree from the database's perspective, we first need to identify the parse tree nodes (words/phrases) that can be mapped to SQL components. Such nodes can be further divided into different types as shown in Figure 4, according to the type of SQL components
$\mathbf{T}$

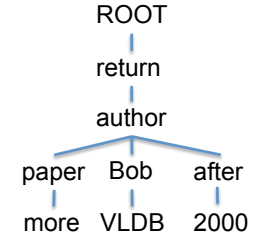

(a)

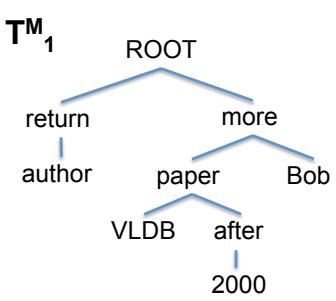

(c)
M return (SN: SELECT) author (NN: author) more (ON: $>$ ) paper (NN: publication) Bob (VN: author.name) VLDB (VN: conference.name) after (ON: >) 2000 (VN: publication.year)

(b)

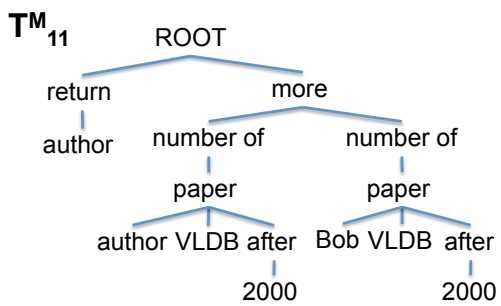

(d)
Figure 3: (a) A Simplified Linguistic Parse Tree from the Stanford Parser. (b) A Mapping Strategy for the Nodes in the Parse Tree. (c) A Valid Parse Tree. (d) A Query Tree after Inserting Implicit Nodes.

\begin{tabular}{|c|l|}
\hline Node Type & \multicolumn{1}{|c|}{ Corresponding SQL Component } \\
\hline Select Node (SN) & SQL keyword: SELECT \\
\hline Operator Node (ON) & an operator, e.g. $=,<=, !=$, contains \\
\hline Function Node (FN) & an aggregation function, e.g., AVG \\
\hline Name Node (NN) & a relation name or attribute name \\
\hline Value Node (VN) & a value under an attribute \\
\hline Quantifier Node (QN) & ALL, ANY, EACH \\
\hline Logic Node (LN) & AND, OR, NOT \\
\hline
\end{tabular}

Figure 4: Different Types of Nodes.

they mapped to. The identification of select node, operator node, function node, quantifier node and logic node is independent of the database being queried. In NaLIR, enumerated sets of phrases are served as the real world "knowledge base" to identify these five types of nodes.

In contrast, name nodes and value nodes correspond to the meta-data and data, respectively, which entirely depend on the database being queried. Often, the words/phrases specified by the user are not exactly the same as the metadata/data in the database. In Section 4.2, we map these parse tree nodes to the meta-data/data in the database based on the similarity evaluation between them. Ambiguity exists when a parse tree node has multiple candidate mappings. In such a case, our system returns multiple candidate mappings for the user to choose from. In Section 4.3, we provide strategies to facilitate the user in recognizing the desired mappings from possibly many candidate mappings. Before that, in Section 4.1, we define the model we assume for the rest of the paper.

\subsection{Data Model}

In this paper, a relation schema is defined as $R_{i}\left(A_{1}^{i}, A_{2}^{i}, \ldots\right.$, $\left.A_{k^{i}}^{i}\right)$, which consists of a relation name $n\left(R_{i}\right)$ and a set of attribute schemas $\left\{A_{j}^{i} \mid 1 \leq j \leq k^{i}\right\}$. A relation schema $R_{i}$ has one (or a set of) attribute schema as its primary key 
$R_{i} . P K$ for identifying the tuples. A database schema $D$ is a set of relation schemas $\left\{R_{i} \mid 1 \leq i \leq n\right\}$. Both relation schemas and attribute schemas are called schema elements. When populated with data, a database schema generates a database and each attribute $A_{j}$ is populated with values $\operatorname{Vals}\left(A_{j}\right)$. In natural language queries, naive users often informally refer to a tuple by specifying the value of one (or a set of) specific attribute. For example, naive users tend to refer to a paper by specifying its title. To capture this intuition, for a relation schema, we may use one (or a set of) attribute $R_{i} . P A$ as its primary attribute to identify tuples informally.

The Schema Graph $G(V, E)$ is a directed graph for a database schema $D$. $V$ consists of two kinds of nodes: relation nodes and attribute nodes, corresponding to relation schemas and attribute schemas in $D$, respectively. Likewise, there are two types of edges in $E$ : projection edges and foreign-key-primary-key (FK-PK) join edges. A projection edge starts from a relation schema $R_{i}$ to each of its attribute schema $A_{1}^{i}, A_{2}^{i}, \ldots, A_{k^{i}}^{i}$, while a FK-PK join edge goes from a foreign key to a primary key when there is a FK-PK relationship between them. For each edge $e$ in $E$, we assign a weight $w(e)$, with a value between 0 and 1 , where a larger weight indicates a stronger connection. A simplified schema graph for the Microsoft Academic Search database is shown in Figure 1, in which some nodes/edges are omitted.

A join path $p$ is a list of schema elements, in which for every two adjacent schema elements $v_{i}$ and $v_{j},\left(v_{i}, v_{j}\right)$ or $\left(v_{j}, v_{i}\right)$ exists in $E$. The weight of $p$ is defined as $w(p)=$ $\Pi_{e_{i} \in p}\left(w\left(e_{i}\right)\right)$. In this paper, join paths containing the following pattern, $p \leftarrow f \rightarrow p$ where $f$ is a foreign key and $p$ is a primary key, are considered as invalid join paths.

\subsection{Candidate Mappings}

In a reasonable design of database schema, the names of schema elements should be meaningful and human-legible. Therefore, when the label of a parse tree node and the name of a schema element are similar in meaning or spelling, they are likely to correspond to the same real world object. To capture this intuition, we use the WUP similarity function [16], denoted as $\operatorname{Sim}_{w}$, which is based on the Wordnet to evaluate the similarity between words/phrases in meaning. In addition, we adopt the square root of the Jaccard Coefficient between the $q$-gram sets of words/phrases, denoted as $\operatorname{Sim}_{q}$, to evaluate their similarity in spelling [17]. Let $l(n)$ be the label of node $n$ and $n(v)$ be the name of a schema element $v$. The name similarity function between $l(n)$ and $n(v)$ is defined as follows:

$\operatorname{Sim}_{n}(l(n), n(v))=\operatorname{MAX}\left\{\operatorname{Sim}_{w}(l(n), n(v)), \operatorname{Sim}_{q}(l(n), n(v))\right\}$

When their similarity is above a predefined threshold $\tau$, we say that $v$ is a candidate mapped schema element of $n$. Also, users may not be able to specify the exact values in the database. In our system, we use $\mathrm{Sim}_{q}$ to evaluate the similarity between the label of a parse tree node and a value. A value is a candidate mapped value of the parse tree node, if their similarity is above $\tau$.

Definition 1 (NV Node). A parse tree node, which has at least one candidate mapped schema element or candidate mapped value, is an $N V$ node.

Since a database often stores meta-data/data closely related to one another, many schema elements/values may be similar to one another, both in meaning and spelling. As a result, multiple candidate mappings may be returned, of which only a subset is correct. For example, in Figure 3 (a), the node "VLDB" may have multiple candidate mappings in the database of Microsoft Academic Search, say, VLDB, VLDB workshops, VLDB PhD workshop, PVLDB and VLDB Journal. In this case, it is very hard for the system to figure out which subset of the candidate mappings the user means.

We deal with this kind of ambiguity interactively with the user. For each ambiguous node, we return multiple of its candidate mappings for the user to choose from. To facilitate the user in recognizing the desired mappings from possibly many candidate mappings, we show candidate mappings hierarchically. In the first level, we show its top $k_{1}$ candidate mapped schema elements or the schema elements containing candidate mapped values. Then, under each of the $k_{1}$ schema elements, we show the top $k_{2}$ candidate mapped values. Users are free to choose a subset of the candidate mapping set as the final mappings. Note that all the final mappings must be of the same type, either in schema element or value. When all the final mappings are schema elements, the node is tokenized as a name node. Otherwise, it will be tokenized as a value node.

Given the vocabulary restriction of the system, some parse tree nodes may fail in mapping to any type of tokens. Also, some words cannot be directly expressed by SQL components. In such a case, a warning is generated, showing the user a list of nodes that do not directly contribute in interpreting the query. Our system deletes each such node from the parse tree and move all its children to its parent.

\subsection{Default Mapping Strategy}

To facilitate user choice, for each node, we would like to choose a mapping as the default mapping, which the user can simply accept in many cases.

A simple solution is to choose the mapping with the highest similarity. But sometimes, we can utilize the structure of the sentence, as reflected in the parse tree, to enhance the quality of the default mapping generation. Consider the query "return all conferences in the database area". The node "database" maps to both the value "database" under Domain.name and the value "database" under Keyword.keyword. Since the node "area" is the parent of the node "database" in the parse tree and maps to Domain with high similarity, the node "database" is more likely to refer to a domain name rather than a keyword. Our intuition is that when NV nodes are closer to each other in the parse tree, which means they are more relevant to each other, they should map to schema elements/values more relevant to each other in the database. The mutual relevance between schema elements is formally defined as follows:

Definition 2 (Relevance Between Schema Elements). Given two schema elements $v_{1}$ and $v_{2}$ in the database, $p\left(v_{1}, v_{2}\right)$ be the join path connecting $v_{1}$ and $v_{2}$ with the highest weight. The weight of $p\left(v_{1}, v_{2}\right)$ is defined as the mutual relevance between $v_{1}$ and $v_{2}$.

When we choose the default mappings for NV nodes, we consider both the similarity in the mappings and the mutual relevance between each pair of NV nodes. We define the score of a mapping strategy below. The mapping strategy 
with the highest score is returned as the default mapping strategy.

Definition 3 (Score of a Mapping Strategy). Let $M$ be a mapping strategy, in which each $N V$ node $n_{i}$ maps to the schema element $v_{n_{i}}$. Let $\left\{\right.$ ancestor $\left.\left(n_{i}, n_{j}\right)\right\}$ be the set of all $N V$ pairs where $n_{i}$ is an ancestor of $n_{j}$ and no $N V$ node exists between $n_{i}$ and $n_{j}$ in the parse tree. The score of $M$ is defined as follows:

$\Pi_{\left\{\operatorname{ancestor}\left(n_{i}, n_{j}\right)\right\}}\left(\operatorname{Sim}\left(n_{i}, v_{n_{i}}\right) * w\left(p\left(v_{i}, v_{j}\right)\right) * \operatorname{Sim}\left(n_{j}, v_{n_{j}}\right)\right)$

\section{PARSE TREE STRUCTURE AJUSTMENT}

Given the correct mapping strategy, each node in the linguistic parse tree can be perfectly understood by our system. In this section, we infer the relationship between the nodes in the linguistic parse tree from the database's perspective and then understand the whole query. However, three obstacles lie in the way of reaching this goal.

First, the linguistic parse tree generated from an off-theshelf parser may be incorrect. Natural language sentences describing complex query logics often have complex structures with modifier attachments, aggregations, comparisons, quantifiers, and conjunctions. As a result, the performance of an off-the-shelf parser is often unsatisfactory for such sentences. For example, the linguistic parse tree shown in Figure 3 (a) is a simplified output of the Stanford Dependency Parser, which incorrectly attaches "VLDB" to "Bob".

Second, the structure of the linguistic parse tree does not directly reflect the relationship between the nodes from the database's perspective. Consider the following three sentence fragments: (a) author who has more than 50 papers, (b) author who has more papers than Bob, and (c) author whose papers are more than Bob. The linguistic parse structures of these three sentence fragments are very different while their semantic meanings are similar from the database's perspective (describing the papers of the author are more than Bob/50). We need to make such relationships explicit and represent them properly.

Third, natural language sentences often contain elliptical expressions. As a result, even though we understand the relationship between all the explicit words/phrases, the sentence may still be ambiguous before the elliptical part is completed. Take the parse tree in Figure 3 (c) as an example. Although the relationship between each pair of nodes is clear, it still has multiple possible interpretations.

In this section, we describe the construction of the Parse Tree Structure Adjustor in detail, which is in charge of correcting the possible errors in the linguistic parse tree, making the relationships between existing nodes understandable from the database's perspective, inserting implicit nodes to the parse tree, and finally obtaining the exact interpretation for the whole query. When ambiguities exist, the parse tree structure adjustor will generate multiple candidate interpretations for the user to choose from.

\subsection{Query Tree}

Since our system is designed to be a query interface that translates natural language queries into SQL statements, the semantic coverage of our system is essentially constrained by the expressiveness of SQL. So, given a database, we represent our semantic coverage as a subset of parse trees, in which each such parse tree explicitly corresponds to a SQL statement and all such parse trees could cover all possible SQL statements (with some constraints). We call such parse trees as Query Trees. As such, interpreting a natural language query (currently represented by a linguistic parse tree and the mapping for each its node) is indeed the process of mapping the query to its corresponding query tree in the semantic coverage.

We defined in Figure 5 the grammar of the parse trees that are syntactically valid in our system (all terminals are different types of nodes defined in Figure 4.). Query trees are the syntactically valid parse trees whose semantic meanings are reasonable, which will be discussed in Section 5.3, or approved by the user. Given the three obstacles in interpreting a linguistic parse tree, as we have discussed before, there is often a big gap between the linguistic parse tree and its corresponding query tree, which makes the mapping between them difficult. In our system, we take the following two strategies to make the mapping process accurate.

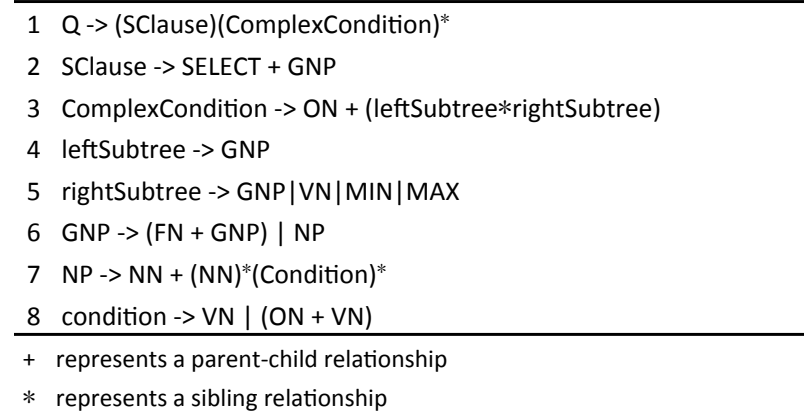

\section{Figure 5: Grammar of Valid Parse Trees.}

First, our system explains a query tree in natural language, which enables the user to verify it. Query trees are intermediates between natural language sentences and SQL statements. Thus the translation from a query tree to a natural language sentence is quite straightforward, compared to that from a SQL statement [9].

Second, given a natural language query, our system will generate multiple candidate query trees for it, which can significantly enhance the probability that one of them is correct. The problem is that, when the query is complex, there may be many candidate query trees, which are similar to each other. To show the user more candidate query trees without burdening them too much in verifying them, we do the mapping in two rounds and communicate with the user after each round. In the first round, we return the top $k$ parse trees, which are syntactically valid according to the grammar defined and can be obtained by only reformulating the nodes in the parse tree. Each such parse tree represents a rough interpretation for the query and we call them valid parse trees. In the second round, implicit nodes (if there is any) are inserted to the chosen (or default) valid parse tree to generate its exact interpretation. Our system inserts implicit nodes one by one under the supervision of the user. In such a way, suppose that there are $k^{\prime}$ possible implicit nodes in each of the $k$ valid parse tree, the user only needs to verify $k$ valid parse trees and $k^{\prime}$ query trees instead of all $k * 2^{k^{\prime}}$ candidate query trees. Figure 3 (c) shows a valid parse tree generated in the first round, while this valid parse tree is full-fledged to the query tree in Figure 3 (d) after inserting implicit nodes. 


\subsection{Parse Tree Reformulation}

In this section, given a linguistic parse tree, we reformulate it in multiple ways and generate its top $k$ rough interpretations. To simplify the tree reformulation process, each logic node or quantifier node is merged with its parent. For example, in the parse tree of Query 3 in Figure 1, which is shown in Figure 7 (a), the quantifier node "each" is merged with its parent "area".

The basic idea in the algorithm is to use subtree move operations to edit the parse tree until it is syntactically valid according to the grammar we defined. The resulting algorithm is shown in Figure 6. Each time, we use the function adjust(tree) to generate all the possible parse trees in one subtree move operation (line 6$)^{2}$. Since the number of possible parse trees grows exponentially with the number of edits, the whole process would be slow. To accelerate the process, our algorithm evaluates each new generated parse tree and filter out bad parse trees directly (line 11 - 12). Also, we hash each parse tree into a number and store all the hashed numbers in a hash table (line 10). By checking the hash table (line 8), we can make sure that each parse tree will be processed at most once. We also set a parameter $t$ as the maximum number of edits approved (line 8). Our system records all the valid parse trees appeared in the reformulation process (line $13-14$ ) and returns the top $k$ of them for the user to choose from (line 15 - 16). Since our algorithm stops after $t$ edits and retains a parse tree only if it is no worse than its corresponding parse tree before the last edit (line 8), some valid parse trees may be omitted.

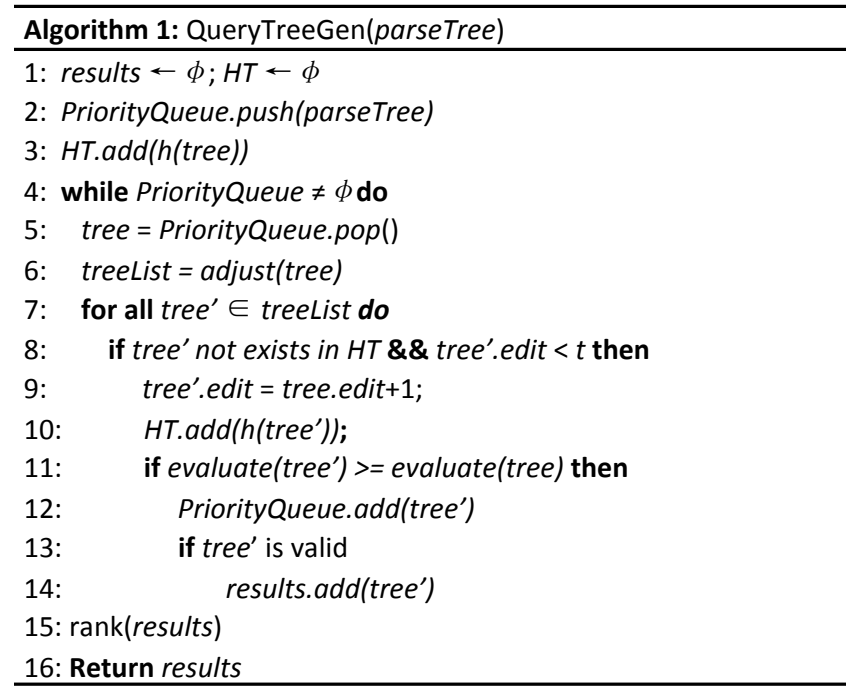

\section{Figure 6: Parse Tree Reformulation Algorithm.}

To filter out bad parse trees in the reformulating process and rank the result parse trees, we evaluate whether a parse tree is desirable from three aspects.

First, a good parse tree should be valid according to the grammar defined in Figure 5. We count the number of parse tree nodes that violate the grammar. The fewer invalid

\footnotetext{
${ }^{2}$ There is an exception for the tree adjustment, in which a node "=" can be inserted to the Root node and the resulting parse tree will be directly added to the priority queue without evaluation.
}

nodes in a parse tree, the better the parse tree is. For example, the parse tree in Figure 3 (a) has four invalid nodes, which are "paper", "more", "Bob" and "VLDB". Similarly, the parse tree in Figure 7 (a) has three invalid nodes: "citation", "most" and "total".

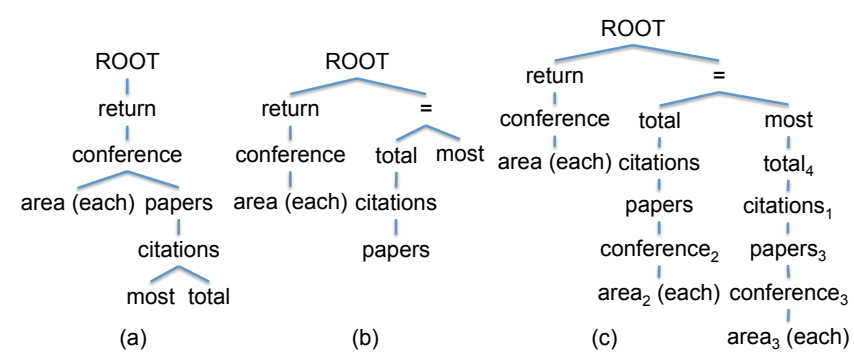

Figure 7: (a) A Simplified Linguistic Parse Tree for Query 3 in Figure 1. (b) A Valid Parse Tree. (c) A Query Tree after Inserting Implicit Nodes.

Second, the mappings between the parse tree nodes and the schema elements in the database can help to infer the desired structure of a parse tree. For example, in the parse tree shown in Figure 3 (a), the node "VLDB" is attached to the node "Bob", which is an incorrect modifier attachment. In the parse tree node mapper, we have mapped the node "VLDB" to Conference.name, "Bob" to Author.name and "paper" to Publication. As discussed in Section 4.3, in the database, Conference.name is more relevant to Publication than to Author.name. So the parse tree, which attaches "VLDB" to "paper", seems more reasonable than the parse tree attaches "VLDB" to "Bob". We capture such intuition by formally defining the score of a parse tree.

Definition 4 (Score of a Parse Tree). Let $T$ be $a$ parse tree, in which each $N V$ node $n t_{i}$ maps to the schema element $v_{i}$. Let $\left\{\operatorname{valid}\left(n t_{i}, n t_{j}\right)\right\}$ be the set of all the $N V$ pairs where $n t_{i}$ is an ancestor of $n t_{j}$ and no $N V$ node exists between $n t_{i}$ and $n t_{j}$. Given the relevance $w\left(p\left(v_{i}, v_{j}\right)\right)$ between $v_{i}$ and $v_{i}$, the score of $T$ is defined as follows:

$$
\operatorname{score}(T)=\Pi_{\left\{\operatorname{valid}\left(n t_{i}, n t_{j}\right)\right\}}\left(w\left(p\left(v_{i}, v_{j}\right)\right)\right)
$$

Third, the parse tree should be similar to the original linguistic parse tree, which is measured by the number of the subtree move operations used in the transformation.

When ranking the all the generated parse trees (line 15 in Figure 6), our system takes all the three factors into account. However, in the tree adjustment process (line 11), to reduce the cases when the adjustments stop in local optima, we only consider the first two factors, in which the first factor dominates the evaluation.

\subsection{Implicit Nodes Insertion}

Natural language sentences often contain elliptical expressions, which make some nodes in their parse trees implicit. In this section, for a rough interpretation, which is represented by a valid parse tree, we obtain its exact interpretation by detecting and inserting implicit nodes.

In our system, implicit nodes mainly exist in complex conditions, which correspond to the conditions involving aggregations, nestings, and non-FKPK join constraints. As can be derived from Figure 5, the semantic meaning of a complex condition is its comparison operator node operating 
on its left and right subtrees. When implicit nodes exist, such syntactically valid conditions are very likely to be semantically "unreasonable". We will first use two examples to illustrate the concept of "unreasonable" conditions and then provide rules to detect and insert the implicit nodes, and finally make "unreasonable" conditions reasonable.

Consider two syntactically valid parse trees, whose semantic meanings are "return all the organizations, where the number of papers by the organization is more than the number of authors in IBM" and "return all the authors, where the number of papers by the author in VLDB is more than the number of papers in ICDE". The first parse tree compares the number of papers with the number of organizations, which sounds unreasonable in meaning. The second parse tree seems better, but compares the number of papers by an author in a conference with all the papers in another conference, which is also a little weird. To detect such unreasonable query logics, we define the concept of core node.

Definition 5 (CORE NODE). Given a complex condition, its left (resp. right) core node is the name node that occurs in its left (right) subtree with no name node as ancestor.

Inspired from [20], given a complex condition, we believe that its left core node and right core node are the concepts that are actually compared. So they should have the same type (map to the same schema element in the database). When they are in different types, we believe that the actual right core node, which is of the same type as the left core node, is implicit. Consider the first query we described above. The left core node is "paper" while the right core node is "author". By inserting an implicit node "paper" as its new right core node, the semantic meaning of the parse tree is changed to "return all the organizations, where the number of papers by the organization is more than the number of papers by the authors in IBM", which is much more reasonable. For the example in Figure 7, the implicit right core node "citation $s_{1}$ " in Figure 7 (c) is inserted in the same way. A special case is that when the right subtree contains only one number, there is no implicit node in the right subtree although the right core node is empty. For example, in the condition "more than 50 papers", the right subtree contains only one number "50" without any implicit node.

The name nodes in a left subtree are always related to the name nodes under the "SELECT" node. Take the parse tree in Figure 7 (b) as an example. The nodes "conference" and "area" are related to the nodes "citations" and "paper". In our system, we connect them by duplicating the name nodes under the "SELECT" node and inserting them to left subtree. Each of the nodes inserted in this step is considered as the same entity with its original node and marked "outside" for the translation in Section 6.

Furthermore, the constraints for the left core node and the right core node should be consistent. Consider the parse tree in Figure 3 (c). Its complex condition compares the number of papers by an author in VLDB after 2000 with the number of all the papers by Bob (in any year on any conference or journal), which is unfair. As such, the constraints of "in VLDB" and "after 2000" should be added to the right subtree. To capture this intuition, we map each NV node under the left core node to at most one NV node under the right core node. Two nodes can be mapped only when they correspond to the same schema element in the database.
When a node has no map, our system will add an implicit node to the other side to make them match with each other. Note that the nodes duplicated from "outside" nodes are also marked "outside" and are considered corresponding to the same entity with the original nodes.

The last kind of implicit node is the function node. In our system, we consider two cases where function nodes may be implicit. First, the function node "count" is often implicit in the natural language sentences. Consider the parse tree in Figure 3 (c). The node "paper" is the left child of node "more" and it maps to the relation "Publication", which is not a number attribute. The comparison between papers is unreasonable without a "count" function. Second, the function nodes operating on the left core node should also operate on the right core node. Figure 7 (c) shows an example for this case. We see that the function node "total" operates on the left core node "citations" but does not operate on the right core node "citations $s_{1}$ ". Our system detects such implicit function node and insert "total 4 " to the right core node.

In our system, the detection and insertion of implicit nodes is just an inference of the semantic meaning for a query, which cannot guarantee the accuracy. As such, the whole process is done under the supervision of the user.

\section{SQL GENERATION}

Given a full-fledged query tree, we show in this section how to generate its corresponding SQL expression.

In the translation, a schema element mapped by an NV node may have multiple representations in the SQL statement, say the schema element itself, its relation, its primary attribute and its primary key. For each occurrence, only one of these representations is adopted according to the context. Take the node "conference" in Figure 7 (c) as an example. When it is added to the FROM clause, it actually refers to the relation Conference. When we return it to the user as the final result, we actually return its primary attribute, which is Conference.name. For simplicity, we use the expression "the schema element mapped by node "conference" in all cases, in which the specific representation can be obtained from the context.

\subsection{Basic Translation}

In the cases when the query tree does not contain function nodes or quantifier nodes, which means the target SQL query will not have aggregate functions or subqueries, the translation is quite straightforward. The schema element mapped by the NV node under the SELECT node is added to the SELECT clause. Each value node (together with its operation node if specified) is translated to a selection condition and added to the WHERE clause. Finally, a FK-PK join path is generated, according to the schema graph, to connect each NV node and its neighbors. Such an FK-PK join path is translated into a series of FK-PK join conditions and all the schema elements in the FK-PK join path are added to the FROM clause.

EXAMPLE 2. Consider the query tree shown in Figure 7 (c). Here we omit its complex condition. The schema element Conference.name, which is mapped by the node "conference", is added to the SELECT clause. To connect the mapped schema elements Conference and Domain, a FK-PK join path Conference $\leftrightarrow$ ConferenceDomain $\leftrightarrow$ Domain is 
generated, which will be translated to the FK-PK conditions shown in line 16-17 in Figure 8.

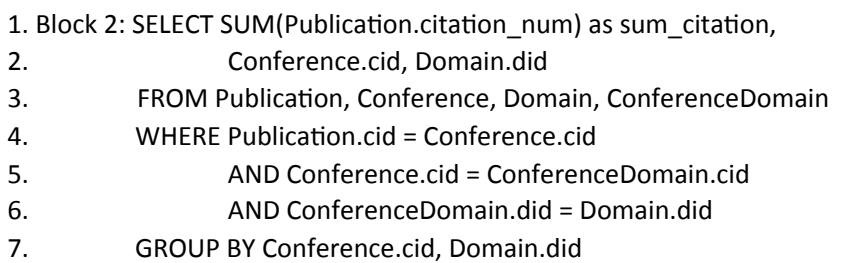

8. Block 3: SELECT MAX(block4.sum citation) as max citation, 9. block4.cid, block4.did

10. FROM (CONTENT OF BLOCK4) as block4

11. GROUP BY block4.did

12.Block 1: SELECT Conference.name, Domain.name

13. FROM Conference, Domain, ConferenceDomain

14. (CONTENT OF BLOCK2) as block2

15. (CONTENT OF BLOCK3) as block3

16. WHERE Conference.cid $=$ ConferenceDomain.cid

17. $\quad$ AND ConferenceDomain. did $=$ Domain. did

18. AND block2.citation_num = block3. max_citation

19. $\quad$ AND Conference.cid $=$ block2.cid

20. $\quad$ AND Conference.cid $=$ block3.cid

21. AND Domain $\cdot$ did $=$ block2 .did

22. $\quad$ AND Domain.did $=$ block3.did

Figure 8: Translated SQL Statement for the Query Tree in Figure 7 (c).

\subsection{Blocks and Subqueries}

When the query tree contains function nodes or quantifier nodes, the target SQL statements will contain subqueries. In our system, we use the concept of block to clarify the scope of each target subquery.

Definition 6 (BLOCK). A block is a subtree rooted at the select node, a name node that is marked "all" or "any", or a function node. The block rooted at the select node is the main block, which will be translated to the main query. Other blocks will be translated to subqueries. When the root of a block $b_{1}$ is the parent of the root of another block $b_{2}$, we say that $b_{1}$ is the direct outer block of $b_{2}$ and $b_{2}$ is a direct inner block of $b_{1}$. The main block is the direct outer block of all the blocks that do not have other outer blocks.

Given a query tree comprising multiple blocks, we translate one block at a time, starting from the innermost block, so that any correlated variables and other context is already set when outer blocks are processed.

EXAMPLE 3. The query tree shown in Figure 7 (c) consists of four blocks: $b_{1}$ rooted at node "return", $b_{2}$ rooted at node "total", $b_{3}$ rooted at node "most", and $b_{4}$ rooted at node "total 4 ". $b_{1}$ is the main block, which is the direct outer block of $b_{2}$ and $b_{3} . b_{3}$ is the direct outer block of $b_{4}$. For this query tree, our system will first translate $b_{2}$ and $b_{4}$, then translate $b_{3}$ and finally translate the main block $b_{1}$.

For each single block, the major part of its translation is the same as the basic translation as we have described. In addition, some SQL fragments must be added to specify the relationship between these blocks.

First, for a block, each of its direct inner blocks is included in the FROM clause as a subquery. Second, each complex condition is translated as a non-FKPK join condition in the main query. Third, each of the name nodes that is marked "outside" refers to the same entity as its original node in the main block. Each of such relationships is translated to a condition in the main query.

EXAMPLE 4. Consider the query tree in Figure 7 (c), whose target $S Q L$ statement is shown in Figure 8 (the block $b_{4}$ is omitted since it is almost the same as $b_{2}$ ). In the query tree, $b_{4}$ is included by $b_{2}$ while $b_{2}$ and $b_{3}$ are included by $b_{1}$ as direct inner blocks. Thus their corresponding subqueries are added to the FROM clause of their direct outer blocks (line 10 and line 14 - 15). The complex condition rooted at node "=" is translated to the condition in line 18. The nodes "conference 2 ", "area a $_{2}$ ", "conference 3 " and "area $a_{3}$ " are marked "outside" in the implicit node insertion, which means they correspond to the same entity as the nodes "conference" and "area" in the main block. These relationships are translated to the conditions in line 19 - 22.

Finally, we need to determine the scope of each aggregate function and quantifier (all, any). The scope of each quantifier is rather obvious, which is the whole block rooted at the name node marked with that quantifier. In contrast, when multiple aggregate functions exist in one query, especially when they occur in different levels of blocks, the scope of each aggregate function is not straightforward. In our system, we call the name node that are marked with "each" or "outside" as grouping nodes. Each aggregate function operates on the grouping nodes that (a) haven't been operated on by other aggregate functions in its inner blocks, and (b) do not have grouping nodes that meet condition (a) as ancestors. Once a grouping node is operated on by an aggregate function, it is disabled (since it has been aggregated). Each time we determine the scope of an aggregate function, we just add all the grouping nodes that still work. Take the query tree in Figure 7 (c) as an example. When determining the scope of total $_{4}$, both the grouping nodes conference 3 and area $_{3}$ work and are added to the GROUP BY clause (the same as line 7). After this aggregation, conference 3 is disabled since it has been operated on by total 4 . When determining the scope of most, only area 3 still works, which will be added to the GROUP BY clause (line 11) and disabled afterwards.

\section{EXPERIMENTS}

We implemented NaLIR as a stand-alone interface that can work on top of any RDBMS. In our implementation, we used MySQL as the RDBMS and the Stanford Natural Language Parser [7] as the dependency parser. For each ambiguity, we limited to 5 the number of interpretations for the user to choose from.

\subsection{Measurement}

The motivation of our system is to enable non-technical users to compose logically complex queries over relational databases and get perfect query results. So, there are two crucial aspects we must evaluate: the quality of the returned results (effectiveness) and whether our system is easy to use for non-technical users (usability). 


\begin{tabular}{|c|c|c|c|}
\hline Relation & \#tuples & Relation & \#tuples \\
\hline Publication & $2.45 \mathrm{M}$ & Author & $1.25 \mathrm{M}$ \\
\hline cite & $20.3 \mathrm{M}$ & Domain & 24 \\
\hline Conference & $2.9 \mathrm{~K}$ & Journal & $1.1 \mathrm{~K}$ \\
\hline Organizations & $11 \mathrm{~K}$ & Keywords & $37 \mathrm{~K}$ \\
\hline
\end{tabular}

Figure 9: Summary Statistics for MAS Database.

Effectiveness. Evaluating the effectiveness of NaLIR is a challenging task. The objective in NaLIR is to allow users to represent SQL statements using natural language. Traditional IR metrics like recall and precision would not work very well since they will always be $100 \%$ if the translated SQL statement is correct and near $0 \%$ in many times when it is not. So, the effectiveness of our system was evaluated as the percentage of the queries that were perfectly answered by our system. (Note that this is a stiff metric, in that we get zero credit if the output SQL query is not perfect, even if the answer set has a high overlap with the desired answer). Since the situations where users accept imperfect/wrong answers would cause severe reliability problems, for the cases when the answers were wrong, we recorded whether the users were able to recognize such failures, whether from the answers themselves or from the explanations generated by our system. Also, for the failure queries, we analyzed the specific reasons that caused such failures.

Usability. For the correctly processed queries, we recorded the actual time taken by the participants. In addition, we evaluated our system subjectively by asking each participant to fill out a post-experiment questionnaire.

\subsection{Experiments Design}

The experiment was a user study, in which participants were asked to finish the query tasks we designed for them.

Data Set and Comparisons. We used the data set of Microsoft Academic Search (MAS). Its simplified schema graph and summary statistics are shown in Figure 1 and Figure 9, respectively. We choosed this data set because it comes with an interesting set of (supported) queries, as we will discuss next.

We compared our system with the faceted interface of the MAS website. The website has a carefully designed ranking system and interface. By clicking through the site, a user is able to get answers to many quite complex queries. We enumerated all query logics that are "directly supported" by the MAS website and can be accomplished by SQL statements. "Directly supported" means that the answer of the query can be obtained in a single webpage (or a series of continuous webpages) without further processing. For example, to answer the query "return the number of conferences in each area", the user has to look at 24 webpages, in which each webpage corresponds to the answer in an area. Thus this query is not considered to be directly supported by the MAS website. However, the query, $Q_{D}$, "return the number of conferences in the Database area" is a directly supported query. Queries that refer to the same relations and attributes but different values, are considered to have the same query logic. Thus, query $Q_{D}$ has the same query logic as the query "return the number of conferences in the Graphics area". Through exhaustive enumeration, we obtained a set of 196 query logics.
Easy: Return all the conferences in database area.

Medium: Return the number of papers in each database conference.

Hard: Return the author who has the most publications in database area.

Figure 10: Sample Queries in Different Complexity.

We marked the complexity of each query according to the levels of aggregation/nesting in its corresponding SQL statement. Sample queries with different complexity are shown in Figure 10. In the query set, the number of easy/medium/hard queries are $63 / 68 / 65$, respectively.

The MAS website is expressly designed to support these 196 query logics, and the user can click through the site to get to a results page, entering only values of constants into search boxes as needed along the way. We used this as the baseline for our comparison. In other words, how did natural language direction compare with click through, for the queries supported by the latter. (Note that an NLIDB supports many different queries beyond just these 196, while the website does not. We restricted our comparison to just the queries supported by the website).

A central innovation in NaLIR is the user interaction as part of query interpretation. To understand the benefit of such interaction, we also experimented with a version of NaLIR in which the interactive communicator was disabled, and the system always chose the default (most likely) option.

Participants. 14 participants were recruited with flyers posted on a university campus. A questionnaire indicated that all participants were familiar with keyword search interfaces (e.g. Google) and faceted search interfaces (e.g. Amazon), but had little knowledge of formal query languages (e.g. SQL). Furthermore, they were fluent in both English and Chinese.

Procedures. We evenly divided the query set into 28 task groups, in which the easy/medium/hard tasks were evenly divided into each task group. This experiment was a within-subject design. Each participant randomly took three groups of tasks and completed three experimental blocks. In the first (resp. second) experimental block, each participant used our system without (with) the Interactive Communicator to accomplish the tasks in her first (second) task group. Then in the third experimental block, each participant used the MAS interface to do her third task group. For each task group, the participants started with sample query tasks, in order to get familiar with each interface.

For our system, it is hard to convey the query task to the participants since any English description would cause bias in the task. To overcome this, we described each query task in Chinese and asked users to compose English query sentences. Since English and Chinese are in entirely different language families, we believe this kind of design can minimize such bias. To alleviate participants' frustration and fatigue from repeated failures, a time limit of three minutes was set for each single query task.

\subsection{Results and Analysis}

Effectiveness. Figure 11 compares the effectiveness of our system (with or without the interactive communicator) with the MAS website. As we can see, when the interactive communicator was disabled, the effectiveness of our system decreased significantly when the query tasks became more complex. Out of the 32 failures, the participants only detected 7 of them. Actually, most of undetected wrong an- 


\begin{tabular}{|l|c|c|c|}
\hline & with Interaction & without Interaction & MAS \\
\hline Simple: & $34 / 34$ & $26 / 32$ & $20 / 33$ \\
\hline Medium: & $34 / 34$ & $23 / 34$ & $18 / 32$ \\
\hline Hard: & $20 / 30$ & $15 / 32$ & $18 / 33$ \\
\hline
\end{tabular}

Figure 11: Effectiveness.

\begin{tabular}{|l|c|c|c|c|}
\hline & Mapper & Reformulation & Insertion & Translation \\
\hline w/o Interaction & 15 & 19 & 0 & 0 \\
\hline with Interaction & 0 & 10 & 0 & 0 \\
\hline
\end{tabular}

Figure 12: Failures in each Component.

swers were aggregated results, which were impossible to verify without further explanation. In other undetected failures, the participants accepted wrong answers mainly because they were not familiar with what they were querying. In the 7 detected failures, although the participants were aware of the failure, they were not able to correctly reformulate the queries in the time constraint. (In 5 of the detected failures, the participants detected the failure only because the query results were empty sets). The situation got much better when the interactive communicator was involved. The users were able to handle 88 out of the 98 query tasks. For the 10 failed tasks, they only accepted 4 wrong answers, which was caused by the ambiguous (natural language) explanations generated from our system. In contrast, the participants were only able to accomplish 56 out of the 98 tasks using the MAS website, although all the correct answers could be found. In the failure cases, the participants were simply not able to find the right webpages, which often required several clicks from the initial search results.

Figure 12 shows the statistics of the specific components that cause the failures. We can see that our system could always correctly detect and insert the implicit parse tree nodes, even without interactive communications with the user. Also, when the query tree was correctly generated, our system translated it to the correct SQL statement. When the interactive communicator was enabled, the accuracy in the parse tree node mapper improved significantly, which means for each the ambiguous parse tree node, the parse tree node mapper could at least generate one correct mapping in the top 5 candidate mappings, and most importantly, the participants were able to recognize the correct mapping from others. The accuracy in parse tree structure reformulation was also improved when the participants were free to choose from the top 5 candidate valid parse trees. However, when the queries were complex, the number of possible valid parse trees was huge. As a result, the top 5 guessed interpretations could not always include the correct one.

Usability. The average time needed for the successfully accomplished query tasks is shown in Figure 13. When the interactive communicator was disabled, the only thing a participant could do was to read the query task description, understand the query task, translate the query task from Chinese to English and submit the query. So most of the query tasks were done in 50 seconds. When the interactive communicator was enabled, the participants were able to read the explanations, choose interpretations, reformulate the query according to the warnings, and decide to whether

\begin{tabular}{|l|c|c|c|}
\hline & with Interaction & without Interaction & MAS \\
\hline Simple: & 48 & 34 & 49 \\
\hline Medium: & 70 & 42 & 67 \\
\hline Hard: & 103 & 51 & 74 \\
\hline
\end{tabular}

Figure 13: Average Time Cost (s).

to accept the query results.

It is worth noting that, using our system (with interactive communicator), there was no instance where the participant became frustrated with the natural language interface and abandoned his/her query task within the time constraint. However, in 9 of the query tasks, participants decided to stop the experiment due to frustration with the MAS website. According to the questionnaire results, the users felt that MAS website was good for browsing data but not well-designed for conducting specific query tasks. They felt NaLIR can handle simple/medium query tasks very well but they encountered difficulties for some of the hard queries. In contrast, the MAS website was not sensitive to the complexity of query tasks. Generally, they welcomed the idea of an interactive natural language query interface, and found our system easy to use. The average level of satisfaction with our system was 5, 5 and 3.8 for easy, medium, and hard query tasks, respectively, on a scale of 1 to 5 , where 5 denotes extremely easy to use.

\section{RELATED WORK}

Keyword search interfaces are widely used by non-experts to specify ad-hoc queries over relational databases [19, 4, $1,8]$. Recently, there has been a stream of such research on keyword search $[15,14,18,5,3]$, in which, given a set of keywords, instead of finding the data relevant to these keywords, they try to interpret the query intent behind the keywords in the view of a formal query language. In particular, some of them extend keywords by supporting aggregation functions [15], Boolean operators [14], query language fragments [5], and so forth. These works can be considered as a first step toward addressing the challenge of natural language querying. Our work builds upon this stream of research. However, our system supports a richer query mechanism that allows us to convey much more complex semantic meaning than a flat collection of keywords.

Natural language interfaces to databases (NLIDB) have been studied for several decades [2]. Early NLIDBs depended on hand crafted semantic grammars tailored to each individual database, which are hard to transport to other databases. In contrast, our system use SQL, a generic query language, as the translation target, which makes our system potentially portable to various databases in different domains.

As pointed out in [13], the major usability problem in NLIDBs is its limited reliability. Natural language sentences do not have formally defined semantics. The goal of NLIDBs is to infer the user's query intent, which cannot guarantee accuracy due to ambiguities. To deal with the reliability issue, PRECISE [13, 12] defines a subset of natural language queries as semantically tractable queries and precisely translates these queries into corresponding SQL queries. However, natural language queries that are not semantically tractable will be rejected by PRECISE. In con- 
trast, our system achieves reliability by explaining to the user how we interpret her query, which is not restricted by the scope of semantically tractable queries.

The idea of interactive NLIDB was discussed in previous literature $[2,10,11]$. Early interactive NLIDBs [2, 10] mainly focus on generating cooperative responses from query results (over-answering). NaLIX [11] takes a step further, generating suggestions for the user to reformulate her query when it is beyond the semantic coverage. This strategy greatly reduces the user's burden in query reformulation. However, the fact that the input query is in the semantic coverage does not necessary mean it will be processed correctly. In our system, given a natural language query, we explain to the user how we process her query and interactively resolve ambiguities with the user. As a result, under the supervision of the user, our system could confidently handle rather complex queries, in which the target SQL statement may include multi-level aggregations, nestings, and various types of joins, among other things.

An important task in our system is to explain the interpretation to the user for verification. Previous systems explain SQL queries to naive users using natural language [9] and visualization tools $[6,3]$. In our system, we explain intermediate logic expressions to the user, which is an easier task.

\section{CONCLUSION AND FUTURE WORK}

We have describe an interactive natural language query interface for relational databases. Given a natural language query, our system first translates it to a SQL statement and then evaluates it against an RDBMS. To achieve high reliability, our system explains to the user how her query is actually processed. When ambiguities exist, for each ambiguity, our system generates multiple likely interpretations for the user to choose from, which resolves ambiguities interactively with the user. The query mechanism described in this paper has been implemented, and actual user experience gathered. Using our system, even naive users are able to accomplish logically complex query tasks, in which the target SQL statements include comparison predicates, conjunctions, quantifications, multi-level aggregations, nestings, and various types of joins, among other things.

In our current implementation, we compose each SQL statement from the ground up. When a query log, which contains natural language queries and their corresponding SQL statements, is available, we believe that our system can benefit from reusing previous SQL statements. When the new query is in the query log, we can directly reuse the existing SQL statement. When the new query is dissimilar to any previous queries, we could compose the SQL statements from the ground up. Most importantly, we would like to achieve somewhere in between, finding similar queries in the query log, reusing some of the SQL fragments, and completing the remaining parts. We believe that such improvements have the potential to enhance the precision of our system, which would reduce the number of interactive communications.

\section{REFERENCES}

[1] S. Agrawal, S. Chaudhuri, and G. Das. Dbxplorer: A system for keyword-based search over relational databases. In ICDE, pages 5-16, 2002.

[2] I. Androutsopoulos, G. D. Ritchie, and P. Thanisch. Natural language interfaces to databases - an introduction. Natural Language Engineering, 1(1):29-81, 1995.

[3] S. Bergamaschi, F. Guerra, M. Interlandi, R. T. Lado, and Y. Velegrakis. Quest: A keyword search system for relational data based on semantic and machine learning techniques. PVLDB, 6(12):1222-1225, 2013.

[4] G. Bhalotia, A. Hulgeri, C. Nakhe, S. Chakrabarti, and S. Sudarshan. Keyword searching and browsing in databases using banks. In ICDE, pages 431-440, 2002.

[5] L. Blunschi, C. Jossen, D. Kossmann, M. Mori, and K. Stockinger. Soda: Generating sql for business users. PVLDB, 5(10):932-943, 2012.

[6] J. Danaparamita and W. Gatterbauer. Queryviz: helping users understand sql queries and their patterns. In EDBT, pages 558-561, 2011.

[7] M.-C. de Marneffe, B. MacCartney, and C. D. Manning. Generating typed dependency parses from phrase structure parses. In $L R E C$, pages 449-454, 2006.

[8] V. Hristidis and Y. Papakonstantinou. Discover: Keyword search in relational databases. In $V L D B$, pages 670-681, 2002.

[9] A. Kokkalis, P. Vagenas, A. Zervakis, A. Simitsis, G. Koutrika, and Y. E. Ioannidis. Logos: a system for translating queries into narratives. In SIGMOD Conference, pages 673-676, 2012.

[10] D. Küpper, M. Strobel, and D. Rösner. Nauda - a cooperative, natural language interface to relational databases. In SIGMOD Conference, pages 529-533, 1993.

[11] Y. Li, H. Yang, and H. V. Jagadish. Nalix: an interactive natural language interface for querying xml. In SIGMOD Conference, pages 900-902, 2005.

[12] A.-M. Popescu, A. Armanasu, O. Etzioni, D. Ko, and A. Yates. Modern natural language interfaces to databases: Composing statistical parsing with semantic tractability. In COLING, 2004.

[13] A.-M. Popescu, O. Etzioni, and H. A. Kautz. Towards a theory of natural language interfaces to databases. In IUI, pages 149-157, 2003.

[14] A. Simitsis, G. Koutrika, and Y. E. Ioannidis. Précis: from unstructured keywords as queries to structured databases as answers. VLDB J., 17(1):117-149, 2008.

[15] S. Tata and G. M. Lohman. Sqak: doing more with keywords. In SIGMOD Conference, pages 889-902, 2008.

[16] Z. Wu and M. S. Palmer. Verb semantics and lexical selection. In $A C L$, pages 133-138, 1994.

[17] C. Xiao, W. Wang, X. Lin, J. X. Yu, and G. Wang. Efficient similarity joins for near-duplicate detection. ACM Trans. Database Syst., 36(3):15, 2011.

[18] D. Xin, Y. He, and V. Ganti. Keyword++: A framework to improve keyword search over entity databases. PVLDB, 3(1):711-722, 2010.

[19] J. X. Yu, L. Qin, and L. Chang. Keyword Search in Databases. Synthesis Lectures on Data Management. Morgan \& Claypool Publishers, 2010.

[20] Y. Zeng, Z. Bao, T. W. Ling, H. V. Jagadish, and G. Li. Breaking out of the mismatch trap. In $I C D E$, pages 940-951, 2014. 